\title{
Essentials of Nursing Care in Randomized Controlled Trials of Nurse-Led Interventions in Somatic Care: A Systematic Review
}

\author{
Bengt Fridlund1,2*, A. C. Jönsson1, E. K. Andersson"1,3, S.-V. Bala1, G.-B. Dahlman'1, \\ A. Forsberg1, S. Glasdam1', A. Hommel1, A. Kristensson'1, C. Lindberg1,3, B. Sivberg1, \\ A. Sjöström-Strand' ${ }^{1}$ J. Wihlborg1, K. Samuelson' ${ }^{1}$ \\ ${ }^{1}$ Department of Health Sciences, Lund University, Lund, Sweden \\ ${ }^{2}$ School of Health Sciences, Jönköping University, Jönköping, Sweden \\ ${ }^{3}$ School of Health Science, Blekinge Institute of Technology, Karlskrona, Sweden \\ Email: ${ }^{*}$ bengt.fridlund@hhj.hj.se
}

Received 16 January 2014; revised 17 February 2014; accepted 1 March 2014

Copyright (C) 2014 by authors and Scientific Research Publishing Inc.

This work is licensed under the Creative Commons Attribution International License (CC BY). http://creativecommons.org/licenses/by/4.0/ (c) (i) Open Access

\section{Abstract}

Background: Nursing practice has to contribute to evidence pointing out why there is a need for more nurse-designed randomized control trials (RCTs) focusing on evidence-based practice (EBP). How far this EBP has progressed in different health aspects is usually established by systematic reviews of RCTs. Nurse-led RCTs exist but no study has addressed the essentials of nursing care. Aim: The aim was therefore to determine the essentials of nurses' interventions by means of nurse-led RCTs in somatic care focusing on the stated context, goals, content, strategies as well as the nurse's role related to effectiveness. Methods: A systematic review was realized according to Cochrane review assumptions to identify, appraise and synthesize all empirical evidence meeting pre-specified eligibility criteria. The PRISMA statement guided the data extraction process $(n=55)$ from PubMed and CINAHL. Results: Of the RCTs in somatic care, $71 \%$ showed a positive effectiveness of nurse-led interventions, of which the nurse had a significant role with regard to being the main responsible in $67 \%$ of the studies. Also, $47 \%$ of the RCTs presented a theoretical standpoint related to the nurse-led interventions and most prominent were international evidence-based guidelines. Goals were found to have either a patient-centered or a professional-centered ambition. Strategies were based on patient-directed initiatives, nurse-patient-directed initiatives or nurse-directed initiatives, while contents were built upon either a patient-nurse interaction or a nursing management plan. Conclusions: This review underlines the necessity of a holistic view of a person, as nurse-led RCTs comprising a patient-centered ambition, patient-directed initiative and patient-nurse interaction plan showed beneficial nursing care effectiveness, particularly if

\footnotetext{
${ }^{*}$ Corresponding author.

How to cite this paper: Fridlund, B., Jönsson, A.C., Andersson, E.K., Bala, S.-V., Dahlman, G.-B., Forsberg, A., Glasdam, S., Kristensson, A., Lindberg, C., Sivberg, B., Sjöström-Strand, A., Wihlborg, J. and Samuelson, K. (2014) Essentials of Nursing Care in Randomized Controlled Trials of Nurse-Led Interventions in Somatic Care: A Systematic Review. Open Journal of Nursing, 4, 181-197. http://dx.doi.org/10.4236/ojn.2014.43023
} 
theory-based. In a nurse-led RCT, a basic theoretical perspective is advantageous as well as to elucidate the role of the nurse in relation to the estimated effects.

\title{
Keywords
}

\author{
Nurse-Led; Nursing Care; Randomized Controlled Trial; Somatic Care; Systematic Review
}

\section{Introduction}

Nursing practice has during the years often been based on experience, tradition and intuition rather than on scientific validation [1]. However, today's healthcare organizations as well as policy-making bodies are emphasizing the importance of evidence-based practice (EBP) [2]. Such EBP has become essential to answering a clinical question, by both awareness of the resources available to nurses and their skills in using them [3]. Still an obvious gap exists between the everyday clinical nursing practice and available empirical evidence about nursing care interventions [4]. Randomized controlled trials (RCTs) are recommended to document the effects of care and treatment [5][6], and in nursing research to evaluate the effectiveness of nursing care interventions [7]. To ascertain the validity and reliability of RCTs, there should be a careful control regarding possible problems, i.e. dropouts, random assignment, identifying and maintaining an adequate control condition, non-adherence to protocols and assessment of clinically meaningful change [8].

Several nursing studies exist aiming to spread knowledge of how to implement EBP, starting with a description of how to search for evidence through the PICOT (Population, Intervention, Comparator, Outcome, Timeframe) format [9], and to form a critical appraisal of the studies available [10]. What seems to be lacking in several RCTs of nursing care interventions is a careful specification how the nursing care has been performed [11]. Few Cochrane reviews exist concerning nurse-led RCTs which also is evident in the somatic care. In a Cochrane review of nursing care interventions such as patient education to improve the ability of self-management, the interventions were often not clearly specified resulting in a conclusion that the evidence did not show effectiveness for the nursing care [12]. In one review concerning RCTs of nursing care interventions for secondary prevention in patients with coronary artery disease, more than half of the trials (57\%) showed positive effectiveness in at least one outcome. However, there were no consistent relationships observed between intervention characteristics and the effectiveness of interventions [13]. This lack of knowledge needs to be developed by establishing not only whether something works, but also why, for whom and in what circumstances [14]. These three aspects could be enlightened by specifying the essentials of nursing care interventions in terms of context, goal, strategy and content in general as well as the significance of the registered nurse's (RN's) role in designing nurse-led RCTs (initiating, organizing, implementing, documenting) in particular. Accordingly, nursing practice has to contribute to evidence and there is an obvious need for more nurse-designed RCTs with focus on EBP [1]. How far this EBP has progressed with reference to the level of evidence in different health aspects is usually established by systematic reviews of RCTs [10]. Results from nurse-led RCTs exist but no study has so far addressed the essentials of nursing care [15]. Consequently, the aim of this systematic review was to determine the essentials of nurses' interventions by means of nurse-led RCTs in somatic care focusing on the stated context, goals, strategies, content as well as the nurse's role related to effectiveness.

\section{Methods}

\subsection{Eligibility Criteria}

In accordance with our aim we included nurse-led RCTs that evaluated the effectiveness of nursing care interventions in the context of somatic care; defining nurse as a RN. To narrow our target area, studies in the field of women's (gynecology/obstetrics), children's (pediatrics) and mental (psychiatric) health were excluded. Participants of interest were patients; hence studies of e.g. relatives were excluded. Outcome measures of interest were patient-reported outcome measurements (PROM) thus excluding studies focusing on e.g. cost analyses and healthcare personnel. 


\subsection{Literature Search}

A review team of 13 nursing researchers, experienced in somatic care performed a literature search in the databases PubMed and CINAHL, with the limits to publication the last five years 2006 to 2010, the English language as the most established international and scientific language, Randomized Control Trials and age group "Adult: 19+ years". In the identification we used both controlled vocabulary (e.g. Medical Subject Headings $[\mathrm{MeSH}])$ and free-text words. The MeSH-terms were "Nurse Clinicians" OR "Nurse Practitioners" and the free-text words were nurse specialist, nurse practitioner, nurse-led and nurse-managed. The literature search also excluded, with the Boolean operator NOT, the following free text words from the search: gynecology, pediatrics, pregnancy and psychiatric.In all; 244 references were found in PubMed and CINAHL and after extracting references already retrieved in PubMed, 207 were left for screening.

\subsection{Study Selection and Data Abstraction}

All retrieved titles and abstracts were screened to determine eligibility. Studies were excluded, if non-RCTs, only study protocols or only pilot studies. After getting full text copies publications were excluded, if non-nurseled, team-led, non-somatic care, non-patient-directed, or non-PROM (Figure 1).
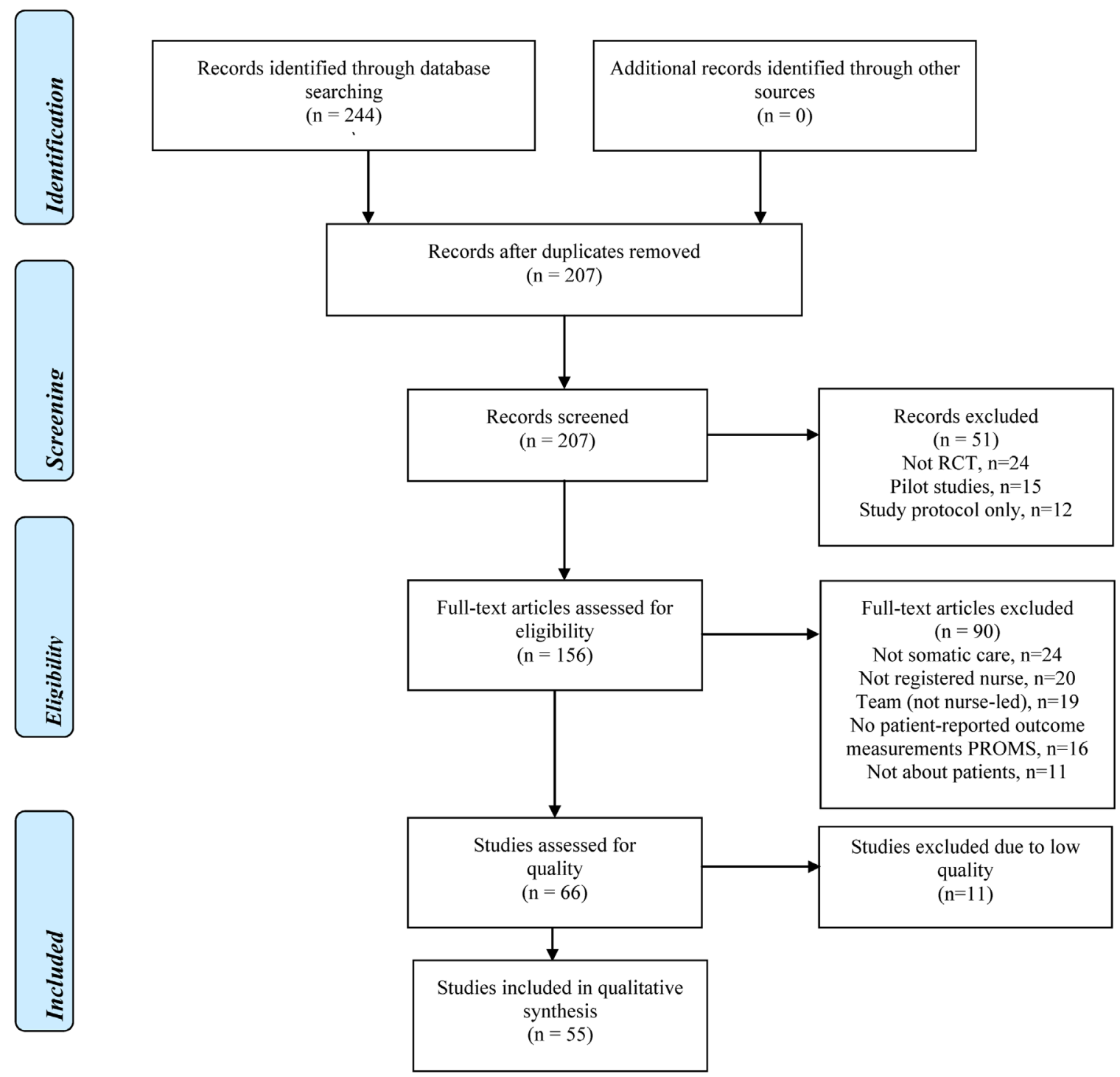

Figure 1. Flow diagram of the systematic review process. 


\subsection{Quality Assessment}

The review team under the direction of first, second and last reviewers abstracted information about and reviewed the publications according to The Swedish Council on Health Technology Assessment's well-established audit template [16]. The following keywords in the audit template were considered: study population, selection criteria, sample size, power calculation, randomization strategy, comparability between groups, blinding, compliance/adherence, primary outcomes, description of intervention and control care and treatment, drop-outs, primary/secondary outcome measures, efficacy/effectiveness, side effects, results, precision, bonds and disqualification. Accordingly, the publications were graded for methodological quality from low through medium to high, the latter indicating a stronger likelihood of the RCT design to generate unbiased results.

\subsection{Data Analysis}

A study protocol inspired by the Preferred Reporting Items for Systematic reviews and Meta-Analyses (PRISMA) statement [17] was used to guide the review team through the data extraction process. The systematic review was then realized according to Cochrane review assumptions [18]; i.e. a transparent and replicable procedure attempting to identify, appraise and synthesize all empirical evidence meeting pre-specified eligibility criteria to answer a given research question. We extracted the following data: context of care, goal, strategy and content, as well as the RN's role based on at least two of four criteria (initiating, organizing, implementing, documenting). The effectiveness was based on the primary outcome stated in the studies. All reviewers scrutinized the extracted data independently followed by review team discussions concerning data quality until consensus was reached.

\section{Results}

\subsection{Demographical and Contextual Data}

As shown in Table 1, over 85\% ( $\mathrm{n}=47)$ of the 55 nurse-led RCTs in somatic care had their origin in Europe (n $=34)$ and North America $(n=13)$; more specific eight European countries were represented, whereas the Netherlands $(n=13)$ and UK $(n=12)$ had prominent positions. In all, four continents were represented; besides Europe and North America, also Asia $(\mathrm{n}=6)$ and Oceania $(\mathrm{n}=2)$. Eight care contexts in somatic care were identified among the 55 RCTs whereas cardiac care $(n=18)$ and primary care $(n=10)$ were the two most prominent ones (Table 1).

\subsection{Goals, Strategies and Content}

Forty-seven percent $(n=26)$ of the RCTs in somatic care presented a theoretical standpoint related to the nurseled intervention (Table 2) and most prominent were international evidence-based guidelines $(\mathrm{n}=9)$. As displayed in Table 3, goals were abstracted into two main categories; a patient-centered ambition and a professional-centered ambition, both comprising almost equal number of categories, i.e. goals. The most prominent goal with the patient-centered ambition was quality of life $(n=11)$ while prevention was the most common goal as to the professional-centered ambition $(n=15)$. Strategies were abstracted into three main categories; patient-directed initiatives, nurse-patient-directed initiatives and nurse-directed initiatives (Table 3). Nurse-patient-directed and nurse-directed initiatives comprehended nearly three times more categories, i.e. strategies than patient-directed initiatives. The most prominent strategy for patient-directed initiatives was video/telemonitoring $(n=4)$ while the corresponding figures for nurse-patient-directed and nurse-directed initiatives were dialogue ( $\mathrm{n}$ $=14$ ) and assessment $(n=17)$, respectively. Contents were abstracted into two main categories (Table 3); a patient-nurse interaction and a nursing management plan, both comprising close equal numbers of categories, i.e. contents. The most prominent content for patient-nurse interaction plan was support and counselling (both, $n=7$ ) while follow-up/feedback $(\mathrm{n}=10)$ was the corresponding content for nursing management plan.

\subsection{The Nurse's Role and Its Effectiveness}

In all, $71 \%$ of the RCTs in somatic care $(n=39)$ showed positive effectiveness of a nurse-led intervention, of which the nurse had had a significant role with regard to being the main responsible in $67 \%(n=26)$ of the studies. Furthermore the theory-based RCTs presented a higher figure (20 of 39; 51\%) with regard to positive ef- 
Table 1. Descriptive overview of the studies included $(n=55)$; context, interventions, effects and role of the nurse.

\begin{tabular}{|c|c|c|c|c|c|c|c|}
\hline \multirow{2}{*}{ Title } & \multirow{2}{*}{$\begin{array}{l}\text { Authors and } \\
\text { country [ref] }\end{array}$} & \multirow{2}{*}{$\begin{array}{l}\text { Context of } \\
\text { care (diagnose } \\
\text { or target } \\
\text { group) }\end{array}$} & \multicolumn{3}{|c|}{ Nurse-led intervention } & \multirow{2}{*}{$\begin{array}{l}\text { Effect based on } \\
\text { primary outcome }\end{array}$} & \multirow{2}{*}{$\begin{array}{l}\text { "Nurs } \\
\text { Sign. }\end{array}$} \\
\hline & & & $\begin{array}{l}\text { Main } \\
\text { Goal }\end{array}$ & $\begin{array}{c}\text { Main } \\
\text { Strategy }\end{array}$ & $\begin{array}{c}\text { Main } \\
\text { Content }\end{array}$ & & \\
\hline $\begin{array}{l}\text { The impact of a } \\
\text { nurse-led care } \\
\text { programme on events } \\
\text { and physical and } \\
\text { psychosocial } \\
\text { parameters in patients } \\
\text { with heart failure with } \\
\text { preserved ejection } \\
\text { fraction: a RCT in } \\
\text { primary care in Russia }\end{array}$ & $\begin{array}{l}\text { Andryukhin } \\
\text { et al. 2010, } \\
\text { Russia [19] }\end{array}$ & $\begin{array}{c}\text { Cardiac } \\
\text { (chronic heart } \\
\text { failure) }\end{array}$ & QoL & $\begin{array}{l}\text { Practical } \\
\text { training }\end{array}$ & Consultation & $\begin{array}{l}\text { Yes, improved } \\
\text { emotional and } \\
\text { physical status, } \\
\text { and QoL }\end{array}$ & No \\
\hline $\begin{array}{c}\text { A randomized } \\
\text { community-based } \\
\text { intervention trial } \\
\text { comparing faith } \\
\text { community nurse } \\
\text { referrals to } \\
\text { telephone-assisted } \\
\text { physician appointments } \\
\text { for health fair } \\
\text { participants with } \\
\text { elevated blood } \\
\text { pressure }\end{array}$ & $\begin{array}{l}\text { Baig et al. } \\
\text { 2007, USA } \\
\text { [20] }\end{array}$ & $\begin{array}{c}\text { Cardiac } \\
\text { (hypertension) }\end{array}$ & Prevention & Assessment & Screening & $\begin{array}{l}\text { No, community nurse } \\
\text { not as effective as } \\
\text { physician (less } \\
\text { reduction in SBP) }\end{array}$ & Yes \\
\hline $\begin{array}{l}{ }^{\mathrm{a}} \text { A RCT to evaluate a } \\
\text { nurse-led programme } \\
\text { of support and lifestyle } \\
\text { management for } \\
\text { patients awaiting } \\
\text { cardiac surgery "Fit for } \\
\text { surgery: Fit for life" } \\
\text { study }\end{array}$ & $\begin{array}{c}\text { Goodman et al. } \\
\text { 2008, UK } \\
\text { [21] }\end{array}$ & $\begin{array}{l}\text { Cardiac } \\
\text { (awaiting } \\
\text { cardiac } \\
\text { surgery) }\end{array}$ & $\begin{array}{l}\text { Self-care } \\
\text { behaviour }\end{array}$ & Dialogue & Counseling & $\begin{array}{l}\text { No effect on anxiety, } \\
\text { LOS, BP, BMI or } \\
\text { S-cholesterol }\end{array}$ & Yes \\
\hline $\begin{array}{l}\text { Improving outcomes } \\
\text { after myocardial } \\
\text { infarction: a } \\
\text { randomized controlled } \\
\text { trial evaluating effects } \\
\text { of a telephone } \\
\text { follow-up intervention }\end{array}$ & $\begin{array}{c}\text { Hanssen et al. } \\
\text { 2007, Norway } \\
\text { [22] }\end{array}$ & $\begin{array}{l}\text { Cardiac } \\
\text { (AMI) }\end{array}$ & QoL & Dialogue & Follow-up & $\begin{array}{l}\text { Yes, improved } \\
\text { HRQoL (physical } \\
\text { dimension) }\end{array}$ & Yes \\
\hline $\begin{array}{l}\text { Effect of moderate or } \\
\text { intensive disease } \\
\text { management program } \\
\text { on outcome in patients } \\
\text { with heart failure: } \\
\text { Coordinating Study } \\
\text { Evaluating Outcomes } \\
\text { of Advising and } \\
\text { Counseling in Heart } \\
\text { Failure (COACH) }\end{array}$ & $\begin{array}{c}\text { Jaarsma et al. } \\
\text { 2008, The } \\
\text { Netherlands } \\
\text { [23] }\end{array}$ & $\begin{array}{l}\text { Cardiac (heart } \\
\text { failure) }\end{array}$ & Adherence & Self-efficacy & Support & $\begin{array}{l}\text { No effect on time } \\
\text { to death or } \\
\text { hospitalization }\end{array}$ & Yes \\
\hline $\begin{array}{l}{ }^{\mathrm{a}} \mathrm{A} \text { nurse-led cardiac } \\
\text { rehabilitation } \\
\text { programme improves } \\
\text { health behaviours and } \\
\text { cardiac physiological } \\
\text { risk parameters: } \\
\text { evidence from } \\
\text { Chengdu, China }\end{array}$ & $\begin{array}{l}\text { Jiang et al. } \\
\text { 2007, China } \\
\text { [24] }\end{array}$ & $\begin{array}{c}\text { Cardiac } \\
\text { (coronary } \\
\text { heart disease) }\end{array}$ & $\begin{array}{l}\text { Self-care } \\
\text { behavior }\end{array}$ & $\begin{array}{l}\text { Adult } \\
\text { learning }\end{array}$ & Support & $\begin{array}{l}\text { Yes, improved } \\
\text { health behavior } \\
\text { and physiological } \\
\text { risk parameters }\end{array}$ & Yes \\
\hline
\end{tabular}




\section{Continued}

\begin{tabular}{|c|c|c|c|c|c|c|c|}
\hline $\begin{array}{l}\text { Surveillance and } \\
\text { treatment of } \\
\text { dyslipidemia in the } \\
\text { post-infarct patient: } \\
\text { can a nurse-led } \\
\text { management approach } \\
\text { make a difference? }\end{array}$ & $\begin{array}{l}\text { Lapointe et al. } \\
\text { 2006, Canada } \\
\text { [25] }\end{array}$ & $\begin{array}{l}\text { Cardiac } \\
\text { (AMI) }\end{array}$ & Adherence & Assessment & Follow-up & $\begin{array}{l}\text { No effect on } \\
\text { lipid profile }\end{array}$ & Yes \\
\hline $\begin{array}{l}{ }^{\mathrm{a}} \text { Effects of a self-care } \\
\text { program on the } \\
\text { health-related quality } \\
\text { of life of pacemaker } \\
\text { patients: a nursing } \\
\text { intervention study }\end{array}$ & $\begin{array}{c}\text { Malm et al. } \\
\text { 2007, Sweden } \\
\text { [26] }\end{array}$ & $\begin{array}{c}\text { Cardiac } \\
\text { (pace maker } \\
\text { patients) }\end{array}$ & QoL & Dialogue & Education & $\begin{array}{l}\text { No effect on } \\
\text { HRQoL }\end{array}$ & Yes \\
\hline $\begin{array}{l}{ }^{\text {a }} \text { Outcomes of cardiac } \\
\text { rehabilitation with } \\
\text { vers- us without a } \\
\text { follow-up intervention } \\
\text { rendered by telephone } \\
\text { (Luebeck follow-up } \\
\text { trial): overall and } \\
\text { gender-specific effects }\end{array}$ & $\begin{array}{c}\text { Mittag et al. } \\
\text { 2006, Germany } \\
\text { [27] }\end{array}$ & $\begin{array}{l}\text { Cardiac } \\
\text { (post cardiac } \\
\text { event) }\end{array}$ & $\begin{array}{l}\text { Self-care } \\
\text { behavior }\end{array}$ & Dialogue & Counseling & $\begin{array}{l}\text { Yes, reduction of } \\
\text { behavioral coronary } \\
\text { risk factors }\end{array}$ & No \\
\hline $\begin{array}{l}\text { aA telephone-delivered } \\
\text { empowerment } \\
\text { intervention with } \\
\text { patients diagnosed } \\
\text { with heart failure }\end{array}$ & $\begin{array}{c}\text { Shearer et al. } \\
\text { 2007, USA } \\
\text { [28] }\end{array}$ & $\begin{array}{c}\text { Cardiac } \\
\text { (heart failure) }\end{array}$ & Self-management & Dialogue & Empowerment & $\begin{array}{l}\text { Yes, facilitated } \\
\text { self-management }\end{array}$ & Yes \\
\hline $\begin{array}{l}\text { Using technology to } \\
\text { create a medication } \\
\text { safety net for cardiac } \\
\text { surgery patients: a } \\
\text { nurse-led RCT }\end{array}$ & $\begin{array}{l}\text { Sherrard et al. } \\
\text { 2009, Canada } \\
\text { [29] }\end{array}$ & $\begin{array}{c}\text { Cardiac } \\
\text { (post-surgery) }\end{array}$ & Adherence & Tele-monitoring & Support & $\begin{array}{c}\text { Yes, increased } \\
\text { compliance } \\
\text { (medication) and } \\
\text { decreased adverse } \\
\text { events }\end{array}$ & Yes \\
\hline $\begin{array}{l}\text { Effects of nurse } \\
\text { management on the } \\
\text { quality of heart failure } \\
\text { care in minority } \\
\text { communities: a } \\
\text { randomized trial }\end{array}$ & $\begin{array}{l}\text { Sisk et al. } \\
\text { 2006, USA } \\
\text { [30] }\end{array}$ & $\begin{array}{c}\text { Cardiac } \\
\text { (ethnically } \\
\text { diverse patients } \\
\text { with heart } \\
\text { failure) }\end{array}$ & Prevention & Assessment & Advice & $\begin{array}{l}\text { Yes, improved } \\
\text { functioning and } \\
\text { fewer } \\
\text { hospitalizations }\end{array}$ & No \\
\hline $\begin{array}{l}{ }^{\mathrm{a}} \text { Nurse-led } \\
\text { self-management } \\
\text { group programme for } \\
\text { patients with } \\
\text { congestive heart } \\
\text { failure: RCT }\end{array}$ & $\begin{array}{c}\text { Smeulders et al. } \\
\text { 2010, The } \\
\text { Netherlands } \\
\text { [31] }\end{array}$ & $\begin{array}{c}\text { Cardiac } \\
\text { (congestive } \\
\text { heart failure) }\end{array}$ & Self-management & it Self-efficacy & Empowerment & $\begin{array}{l}\text { No effect on } \\
\text { psychosocial } \\
\text { attributes, self-care } \\
\text { behavior or QoL }\end{array}$ & Yes \\
\hline $\begin{array}{c}{ }^{\mathrm{a}} \text { Effectsof a } \\
\text { post-discharge } \\
\text { transitional care } \\
\text { programme for } \\
\text { patients with coronary } \\
\text { heart disease in China: } \\
\text { a RCT }\end{array}$ & $\begin{array}{c}\text { Zhao and Wong } \\
\text { 2009, China } \\
\text { [32] }\end{array}$ & $\begin{array}{l}\text { Cardiac } \\
\text { (coronary heart } \\
\text { disease) }\end{array}$ & t Self-management & Self-efficacy & Empowerment & $\begin{array}{l}\text { Yes, positive effect } \\
\text { on diet, medication } \\
\text { and health-related } \\
\text { life-style }\end{array}$ & Yes \\
\hline $\begin{array}{l}\text { Nurse practitioners } \\
\text { substituting for } \\
\text { general practitioners: } \\
\text { RCT }\end{array}$ & $\begin{array}{c}\text { Dierick-van } \\
\text { Daele et al. } \\
\text { 2009, The } \\
\text { Netherlands } \\
\text { [33] }\end{array}$ & $\begin{array}{c}\text { Primary } \\
\text { (primary care } \\
\text { patients) }\end{array}$ & $\begin{array}{c}\text { Patient } \\
\text { satisfaction }\end{array}$ & Assessment & Consultation & $\begin{array}{l}\text { Yes, NP as effective } \\
\text { as GP (patient } \\
\text { satisfaction with } \\
\text { care) }\end{array}$ & Yes \\
\hline $\begin{array}{c}\text { Larval therapy for leg } \\
\text { ulcers (VenUS II): } \\
\text { RCT }\end{array}$ & $\begin{array}{c}\text { Dumville et al. } \\
\text { 2009, UK } \\
\text { [34] }\end{array}$ & $\begin{array}{l}\text { Primary } \\
\text { (leg ulcer) }\end{array}$ & EBC & Assessment & Wound care & $\begin{array}{l}\text { No effect on rate of } \\
\text { healing }\end{array}$ & Yes \\
\hline
\end{tabular}




\section{Continued}

\begin{tabular}{|c|c|c|c|c|c|c|c|}
\hline $\begin{array}{l}{ }^{\mathrm{a}} \text { Effectiveness of a } \\
\text { falls-and-fracture nurse } \\
\text { coordinator to reduce } \\
\text { falls: a RCT of at-risk } \\
\text { older adults }\end{array}$ & $\begin{array}{l}\text { Elley et al. } \\
\text { 2008, New } \\
\text { Zeeland [35] }\end{array}$ & $\begin{array}{c}\text { Primary } \\
\text { (elderly } \\
\text { identified for } \\
\text { falls) }\end{array}$ & Prevention & Assessment & Screening & $\begin{array}{c}\text { No effect on } \\
\text { proportion of falls }\end{array}$ & Yes \\
\hline $\begin{array}{l}{ }^{\mathrm{a}} \text { Randomized clinical } \\
\text { effectiveness trial of } \\
\text { nurse-administered } \\
\text { small-group cognitive } \\
\text { behavior therapy for } \\
\text { persistent insomnia } \\
\text { in general practice }\end{array}$ & $\begin{array}{c}\text { Espie et al. } \\
\text { 2007, UK } \\
{[36]}\end{array}$ & $\begin{array}{l}\text { Primary } \\
\text { (persistent } \\
\text { insomnia) }\end{array}$ & EBC & CBT & Consultation & Yes, improved sleep & No \\
\hline $\begin{array}{l}\text { *Nurse clinic versus } \\
\text { home delivery of } \\
\text { evidence-based } \\
\text { community leg ulcer } \\
\text { care: a randomized } \\
\text { health services trial }\end{array}$ & $\begin{array}{c}\text { Harrison et al. } \\
\text { 2008, Canada } \\
\text { [37] }\end{array}$ & $\begin{array}{l}\text { Primary } \\
\text { (leg ulcer) }\end{array}$ & EBC & EBC algorithm & Wound care & $\begin{array}{c}\text { Yes, clinic delivery } \\
\text { of care as effective } \\
\text { as home delivery } \\
\text { (3 months healing } \\
\text { rate) }\end{array}$ & Yes \\
\hline $\begin{array}{c}{ }^{\mathrm{a}} \text { A minimal } \\
\text { psychological } \\
\text { intervention in } \\
\text { chronically ill elderly } \\
\text { patients with } \\
\text { depression: } \\
\text { a randomized trial }\end{array}$ & $\begin{array}{c}\text { Lamers et al. } \\
\text { 2010a, The } \\
\text { Netherlands } \\
\text { [38] }\end{array}$ & $\begin{array}{c}\text { Primary } \\
\text { (chronically ill } \\
\text { elderly with } \\
\text { depression) }\end{array}$ & Self-management & CBT & Counseling & $\begin{array}{l}\text { Yes, reduced } \\
\text { symptoms of } \\
\text { depression }\end{array}$ & No \\
\hline $\begin{array}{l}\text { The Winchester falls } \\
\text { project: a RCT of } \\
\text { secondary prevention } \\
\text { of falls in older people }\end{array}$ & $\begin{array}{c}\text { Spice et al. } \\
\text { 2009, UK } \\
{[39]}\end{array}$ & $\begin{array}{l}\text { Primary } \\
\text { (elderly } \\
\text { identified } \\
\text { for falls) }\end{array}$ & Detection & Assessment & Screening & $\begin{array}{c}\text { No effect on } \\
\text { proportion of falls }\end{array}$ & Yes \\
\hline $\begin{array}{l}{ }^{\text {a }} \text { Preventing weight } \\
\text { gain: one-year results } \\
\text { of a randomized } \\
\text { lifestyle intervention }\end{array}$ & $\begin{array}{c}\text { ter Bogt et al. } \\
\text { 2009, The } \\
\text { Netherlands } \\
\text { [40] }\end{array}$ & $\begin{array}{c}\text { Primary } \\
\text { (overweight } \\
\text { or obesity) }\end{array}$ & Prevention & Dialogue & Counseling & $\begin{array}{l}\text { Yes, reduced body } \\
\text { weight }\end{array}$ & Yes \\
\hline $\begin{array}{l}\text { Effect of an intensive } \\
\text { nurse-managed medical } \\
\text { care programme on } \\
\text { ambulatory blood } \\
\text { pressure in } \\
\text { hypertensive patients }\end{array}$ & $\begin{array}{c}\text { Ulm et al. } \\
\text { 2010, Germany } \\
\text { [41] }\end{array}$ & $\begin{array}{c}\text { Primary } \\
\text { (hypertension) }\end{array}$ & Prevention & Self-monitoring & Advice & $\begin{array}{l}\text { Yes, decline of } \\
\text { systolic BP }\end{array}$ & No \\
\hline $\begin{array}{l}\text { Nurse led, home based } \\
\text { self help treatment for } \\
\text { patients in primary care } \\
\text { with chronic fatigue } \\
\text { syndrome: RCT }\end{array}$ & $\begin{array}{l}\text { Wearden et al. } \\
\text { 2010, UK } \\
\text { [42] }\end{array}$ & $\begin{array}{c}\text { Primary } \\
\text { (chronic fatigue } \\
\text { syndrome) }\end{array}$ & QoL & $\begin{array}{l}\text { Listening } \\
\text { therapy }\end{array}$ & Support & $\begin{array}{c}\text { No effect on fatigue } \\
\text { or physical } \\
\text { functioning }\end{array}$ & Yes \\
\hline $\begin{array}{c}{ }^{a} \text { Managing uncertainty } \\
\text { in diabetes: an } \\
\text { intervention for } \\
\text { older African } \\
\text { American women }\end{array}$ & $\begin{array}{l}\text { Amoako and } \\
\text { Skelly } \\
\text { 2007, USA } \\
{[43]}\end{array}$ & $\begin{array}{c}\text { Diabetes } \\
\text { (Afro American } \\
\text { women with } \\
\text { diabetes) }\end{array}$ & Self-management & Dialogue & Counseling & $\begin{array}{l}\text { Yes, reduction of } \\
\text { diabetes-related } \\
\text { uncertainty }\end{array}$ & Yes \\
\hline $\begin{array}{l}{ }^{\mathrm{a}} \text { Improving glycemic } \\
\text { control in older adults } \\
\text { using a videophone } \\
\text { motivational diabetes } \\
\text { self-management } \\
\text { intervention }\end{array}$ & $\begin{array}{c}\text { Hawkins } \\
\text { 2010, USA } \\
{[44]}\end{array}$ & $\begin{array}{c}\text { Diabetes } \\
\text { (uncontrolled } \\
\text { diabetic adults) }\end{array}$ & Self-management & Self-efficacy & Empowerment & $\begin{array}{c}\text { Yes, decreased } \\
\text { HBA1c }\end{array}$ & Yes \\
\hline $\begin{array}{l}{ }^{\mathrm{a}} \text { Diabetes specialist } \\
\text { nurse as main care } \\
\text { provider for patients } \\
\text { with type } 2 \text { diabetes }\end{array}$ & $\begin{array}{c}\text { Houweling et al. } \\
\text { 2009, The } \\
\text { Netherlands } \\
\text { [45] }\end{array}$ & $\begin{array}{l}\text { Diabetes (type } \\
2 \text { diabetes) }\end{array}$ & EBC & EBC algoritm & Consultation & $\begin{array}{c}\text { Yes, CNS as effective } \\
\text { as internist (HBA1c } \\
\text { decrease) }\end{array}$ & Yes \\
\hline
\end{tabular}




\section{Continued}

\begin{tabular}{|c|c|c|c|c|c|c|c|}
\hline $\begin{array}{l}\text { Preventing glycaemic } \\
\text { relapse in recently } \\
\text { controlled type } 2 \\
\text { diabetes patients: } \\
\text { a RCT }\end{array}$ & $\begin{array}{c}\text { Huizinga et al. } \\
\text { 2010, USA } \\
{[46]}\end{array}$ & $\begin{array}{l}\text { Diabetes (type } \\
2 \text { diabetes) }\end{array}$ & Prevention & Dialogue & Support & $\begin{array}{l}\text { No effect on } \\
\text { HBA1c }\end{array}$ & Yes \\
\hline $\begin{array}{l}\text { A nurse short message } \\
\text { service by cellular } \\
\text { phone in type- } 2 \text { diabetic } \\
\text { patients for six months }\end{array}$ & $\begin{array}{c}\text { Kim and Jeong } \\
\text { 2007, South } \\
\text { Korea } \\
\text { [47] }\end{array}$ & $\begin{array}{l}\text { Diabetes (type } \\
2 \text { diabetes) }\end{array}$ & EBC & Self-monitoring & Advice & $\begin{array}{l}\text { Yes, improved } \\
\text { HBA1c }\end{array}$ & Yes \\
\hline $\begin{array}{l}\text { An intensive nurse-led, } \\
\text { multi-interventional } \\
\text { clinic is more } \\
\text { successful in achieving } \\
\text { vascular risk reduction } \\
\text { targets than standard } \\
\text { diabetes care }\end{array}$ & $\begin{array}{l}\text { MacMahonTone } \\
\text { et al. } \\
\text { 2009, Ireland } \\
\text { [48] }\end{array}$ & $\begin{array}{l}\text { Diabetes (type } \\
2 \text { diabetes) }\end{array}$ & Prevention & Assessment & Support & $\begin{array}{l}\text { Yes, reduction in } \\
\text { vascular risk }\end{array}$ & No \\
\hline $\begin{array}{l}\text { Effect of telephone } \\
\text { follow-up on adherence } \\
\text { to a diabetes therapeutic } \\
\text { regimen }\end{array}$ & $\begin{array}{c}\text { Nesari et al. } \\
\text { 2010, Iran } \\
\text { [49] }\end{array}$ & $\begin{array}{l}\text { Diabetes (type } \\
2 \text { diabetes) }\end{array}$ & Adherence & Dialogue & Follow-up & $\begin{array}{c}\text { Yes, decreased } \\
\text { HBA1c }\end{array}$ & Yes \\
\hline $\begin{array}{c}\text { Active care } \\
\text { management supported } \\
\text { by home telemonitoring } \\
\text { in veterans with type } 2 \\
\text { diabetes: the DiaTel } \\
\text { RCT }\end{array}$ & $\begin{array}{c}\text { Stone et al. } \\
\text { 2010, USA } \\
{[50]}\end{array}$ & $\begin{array}{c}\text { Diabetes } \\
\text { (veterans with } \\
\text { type } 2 \text { diabetes) }\end{array}$ & Adherence & Tele-monitoring & Follow-up & $\begin{array}{l}\text { Yes, decreased } \\
\text { HBA1c }\end{array}$ & Yes \\
\hline $\begin{array}{c}\text { A RCT of nurse-led } \\
\text { care for symptomatic } \\
\text { moderate-severe } \\
\text { obstructive sleep } \\
\text { apnea }\end{array}$ & $\begin{array}{c}\text { Antic et al. } \\
\text { 2009, Australia } \\
\text { [51] }\end{array}$ & $\begin{array}{l}\text { Respiratory } \\
\text { (obstructive } \\
\text { sleep apnea) }\end{array}$ & QoL & Assessment & Consultation & $\begin{array}{c}\text { Yes, care model as } \\
\text { effective as physician } \\
\text { directed care (daytime } \\
\text { sleepiness) }\end{array}$ & Yes \\
\hline $\begin{array}{l}{ }^{\text {a} I m p r o v i n g ~ q u a l i t y ~ o f ~} \\
\text { life in depressed COPD } \\
\text { patients: effectiveness } \\
\text { of a minimal } \\
\text { psychological } \\
\text { intervention }\end{array}$ & $\begin{array}{l}\text { Lamers et al. } \\
\text { 2010b, The } \\
\text { Netherlands } \\
\text { [52] }\end{array}$ & $\begin{array}{l}\text { Respiratory } \\
\text { (COPD and } \\
\text { depressive } \\
\text { symptoms) }\end{array}$ & Self-management & CBT & Counseling & $\begin{array}{l}\text { Yes, reduced } \\
\text { symptoms of anxiety } \\
\text { and depression, } \\
\text { improved QoL }\end{array}$ & No \\
\hline $\begin{array}{l}\text { A RCT of follow-up } \\
\text { of patients discharged } \\
\text { from the hospital } \\
\text { following acute asthma: } \\
\text { best performed by } \\
\text { specialist nurse or } \\
\text { doctor? }\end{array}$ & $\begin{array}{c}\text { Nathan et al. } \\
\text { 2006, UK } \\
{[53]}\end{array}$ & $\begin{array}{l}\text { Respiratory } \\
\text { (post-acute } \\
\text { asthma) }\end{array}$ & Prevention & Assessment & Follow-up & $\begin{array}{l}\text { Yes, CNS as effective } \\
\text { as respiratory doctor } \\
\text { (number of } \\
\text { exacerbations) }\end{array}$ & Yes \\
\hline $\begin{array}{c}\text { A nurse led } \\
\text { intermediate care } \\
\text { package in patients who } \\
\text { have been hospitalised } \\
\text { with an acute } \\
\text { exacerbation of chronic } \\
\text { obstructive pulmonary } \\
\text { disease }\end{array}$ & $\begin{array}{l}\text { Sridhar et al. } \\
\text { 2008, UK } \\
\text { [54] }\end{array}$ & $\begin{array}{l}\text { Respiratory } \\
\text { (post-acute } \\
\text { exacerbation of } \\
\text { COPD) }\end{array}$ & Prevention & Care plan & Education & $\begin{array}{l}\text { No effect on hospital } \\
\text { admission rate }\end{array}$ & Yes \\
\hline $\begin{array}{l}\text { Weekly self-monitoring } \\
\text { and treatment } \\
\text { adjustment benefit } \\
\text { patients with partly } \\
\text { controlled and } \\
\text { uncontrolled asthma: } \\
\text { an analysis of the } \\
\text { SMASHING study }\end{array}$ & $\begin{array}{l}\text { van der Meer } \\
\text { et al. } \\
\text { 2010, The } \\
\text { Netherlands } \\
\text { [55] }\end{array}$ & $\begin{array}{l}\text { Respiratory } \\
\text { (asthma) }\end{array}$ & Self-management & Self-monitoring & Advice & $\begin{array}{l}\text { Yes, improved } \\
\text { asthma control }\end{array}$ & Yes \\
\hline
\end{tabular}




\section{Continued}

\begin{tabular}{|c|c|c|c|c|c|c|c|}
\hline $\begin{array}{c}\text { Tele-assistance in } \\
\text { chronic respiratory } \\
\text { failure patients: } \\
\text { a RCT }\end{array}$ & $\begin{array}{c}\text { Vitacca et al. } \\
\text { 2009, Italy } \\
{[56]}\end{array}$ & $\begin{array}{l}\text { Respiratory } \\
\text { (COPD) }\end{array}$ & Prevention & Assessment & Consultation & $\begin{array}{c}\text { Yes, fewer } \\
\text { hospitalizations }\end{array}$ & No \\
\hline $\begin{array}{l}{ }^{\text {a }} \text { The effectiveness of } \\
\text { nurse-led } \\
\text { telemonitoring of } \\
\text { asthma: results of a } \\
\text { RCT }\end{array}$ & $\begin{array}{l}\text { Willems et al. } \\
\text { 2008, The } \\
\text { Netherlands } \\
\text { [57] }\end{array}$ & $\begin{array}{l}\text { Respiratory } \\
\text { (asthma) }\end{array}$ & QoL & Tele-monitoring & $\begin{array}{c}\text { Symptom } \\
\text { management }\end{array}$ & $\begin{array}{c}\text { No effect on } \\
\text { asthma-specific } \\
\text { QoL }\end{array}$ & Yes \\
\hline $\begin{array}{c}\text { The PRaCTICaL study } \\
\text { of nurse led, intensive } \\
\text { care follow-up } \\
\text { programmes for } \\
\text { improving long term } \\
\text { outcomes from critical } \\
\text { illness: a pragmatic } \\
\text { RCT }\end{array}$ & $\begin{array}{l}\text { Cuthbertson } \\
\text { et al. } \\
\text { 2009, UK } \\
\text { [58] }\end{array}$ & $\begin{array}{c}\text { Acute } \\
\text { (intensive } \\
\text { care patients) }\end{array}$ & QoL & Dialogue & Follow-up & $\begin{array}{l}\text { No effect on } \\
\text { HRQoL }\end{array}$ & Yes \\
\hline $\begin{array}{l}\text { Injured road users’ } \\
\text { health-related quality } \\
\text { of life after telephone } \\
\text { intervention: a RCT }\end{array}$ & $\begin{array}{c}\text { Franzén et al. } \\
\text { 2009, Sweden } \\
\text { [59] }\end{array}$ & $\begin{array}{l}\text { Acute (injured } \\
\text { road users) }\end{array}$ & QoL & Dialogue & Advice & $\begin{array}{l}\text { Yes, increased } \\
\text { HRQoL }\end{array}$ & Yes \\
\hline $\begin{array}{l}\text { Infection, rejection, } \\
\text { and hospitalizations in } \\
\text { transplant recipients } \\
\text { using telehealth }\end{array}$ & $\begin{array}{c}\text { Leimig et al. } \\
\text { 2008, USA } \\
{[60]}\end{array}$ & $\begin{array}{c}\text { Acute } \\
\text { (transplant } \\
\text { recipients) }\end{array}$ & Detection & Dialogue & $\begin{array}{c}\text { Symptom } \\
\text { management }\end{array}$ & $\begin{array}{c}\text { Yes, as effective } \\
\text { as standard care } \\
\text { (infection, rejection } \\
\text { and hospitalization) }\end{array}$ & Yes \\
\hline $\begin{array}{l}\text { Randomized trial of a } \\
\text { delirium abatement } \\
\text { program for postacute } \\
\text { skilled nursing facilities }\end{array}$ & $\begin{array}{l}\text { Marcantonio } \\
\text { et al. } \\
\text { 2010, USA } \\
{[61]}\end{array}$ & $\begin{array}{l}\text { Acute (elderly } \\
\text { with acute } \\
\text { illness) }\end{array}$ & Prevention & Assessment & $\begin{array}{c}\text { Symptom } \\
\text { management }\end{array}$ & $\begin{array}{l}\text { No effect on } \\
\text { persistence of } \\
\text { delirium }\end{array}$ & Yes \\
\hline $\begin{array}{c}{ }^{\mathrm{a}} \text { Effects of } \\
\text { nurse-managed } \\
\text { telemonitoring on blood } \\
\text { pressure at 12-month } \\
\text { follow-up among urban } \\
\text { African Americans }\end{array}$ & $\begin{array}{c}\text { Artinian et al. } \\
\text { 2007, USA } \\
\text { [62] }\end{array}$ & $\begin{array}{l}\text { Cardiovascular } \\
\text { (Afro } \\
\text { Americans } \\
\text { with } \\
\text { hypertension) }\end{array}$ & Prevention & Tele-monitoring & Feedback & $\begin{array}{l}\text { Yes, improved BP } \\
\text { control (reduction } \\
\text { in SBP) }\end{array}$ & Yes \\
\hline $\begin{array}{c}{ }^{\mathrm{a}} \text { A randomized, } \\
\text { controlled trial for risk } \\
\text { factor reduction in } \\
\text { patients with } \\
\text { symptomatic vascular } \\
\text { disease: the } \\
\text { multidisciplinary } \\
\text { Vascular Prevention by } \\
\text { Nurses Study (VENUS) }\end{array}$ & $\begin{array}{c}\text { Goessens et al. } \\
\text { 2006, The } \\
\text { Netherlands } \\
\text { [63] }\end{array}$ & $\begin{array}{l}\text { Cardiovascular } \\
\text { (symptomatic } \\
\text { vascular } \\
\text { disease) }\end{array}$ & Prevention & Care plan & Support & $\begin{array}{c}\text { Yes, improved } \\
\text { cardiovascular risk } \\
\text { profile }\end{array}$ & Yes \\
\hline $\begin{array}{l}{ }^{\mathrm{a}} \text { The role of } \\
\text { self-efficacy in vascular } \\
\text { risk factor management: } \\
\text { a randomized controlled } \\
\text { trial }\end{array}$ & $\begin{array}{c}\text { Sol et al. } \\
\text { 2007, The } \\
\text { Netherlands } \\
\text { [64] }\end{array}$ & $\begin{array}{l}\text { Cardiovascular } \\
\text { (symptomatic } \\
\text { vascular } \\
\text { disease) }\end{array}$ & $\begin{array}{l}\text { Self-care } \\
\text { behavior }\end{array}$ & Self-efficacy & Empowerment & $\begin{array}{l}\text { No effect on } \\
\text { self-efficacy }\end{array}$ & Yes \\
\hline $\begin{array}{l}{ }^{\mathrm{a}} \text { Effect of nurse } \\
\text { counselling on } \\
\text { metabolic risk factors } \\
\text { in patients with mild } \\
\text { hypertension: a } \\
\text { randomised controlled } \\
\text { trial }\end{array}$ & $\begin{array}{c}\text { Tonstad et al. } \\
\text { 2007, Norway } \\
{[65]}\end{array}$ & $\begin{array}{l}\text { Cardiovascular } \\
\text { (hypertension) }\end{array}$ & $\begin{array}{l}\text { Self-care } \\
\text { behavior }\end{array}$ & Dialogue & Counseling & $\begin{array}{l}\text { Yes, improved } \\
\text { weight control and } \\
\text { lower triglycerides }\end{array}$ & No \\
\hline
\end{tabular}




\section{Continued}

\begin{tabular}{|c|c|c|c|c|c|c|c|}
\hline $\begin{array}{l}\text { A RCT of structured } \\
\text { nurse-led outpatient } \\
\text { clinic follow-up for } \\
\text { dyspeptic patients } \\
\text { after direct access } \\
\text { gastroscopy }\end{array}$ & $\begin{array}{l}\text { Chan et al. } \\
\text { 2009, UK } \\
{[66]}\end{array}$ & $\begin{array}{c}\text { Medical } \\
\text { (dyspepsia } \\
\text { after } \\
\text { gastroscopy) }\end{array}$ & Prevention & Assessment & Follow-up & $\begin{array}{l}\text { Yes, NP as effective } \\
\text { as GP (dyspepsia } \\
\text { severity, HRQoL, } \\
\text { drug use and cost) }\end{array}$ & No \\
\hline $\begin{array}{l}{ }^{\mathrm{a}} \text { Health-related quality } \\
\text { of life in patients } \\
\text { undergoing peritoneal } \\
\text { dialysis: effects of a } \\
\text { nurse-led case } \\
\text { management } \\
\text { programme }\end{array}$ & $\begin{array}{c}\text { Chow \& Wong } \\
\text { 2010, Hong } \\
\text { Kong } \\
{[67]}\end{array}$ & $\begin{array}{c}\text { Medical } \\
\text { (peritoneal or } \\
\text { hemo-dialysis) }\end{array}$ & QoL & Care plan & Motivation & $\begin{array}{l}\text { Yes, improved QoL } \\
\text { (within group } \\
\text { differences over } \\
\text { time) }\end{array}$ & Yes \\
\hline $\begin{array}{l}\text { Telephonic } \\
\text { management of rectal } \\
\text { bleeding in young } \\
\text { adults: a prospective } \\
\text { RCT }\end{array}$ & $\begin{array}{l}\text { Raje et al. } \\
\text { 2007, UK } \\
{[68]}\end{array}$ & $\begin{array}{c}\text { Medical (rectal } \\
\text { bleeding) }\end{array}$ & Prevention & Assessment & Advice & $\begin{array}{l}\text { Yes, symptomatic } \\
\text { improvement } \\
\text { (resolution of rectal } \\
\text { bleedings) }\end{array}$ & No \\
\hline $\begin{array}{l}{ }^{\mathrm{a}} \text { Evaluation of a } \\
\text { nurse-led disease } \\
\text { management } \\
\text { programme for } \\
\text { chronic kidney } \\
\text { disease: a RCT }\end{array}$ & $\begin{array}{l}\text { Wong et al. } \\
\text { 2010, Hong } \\
\text { Kong } \\
\text { [69] }\end{array}$ & $\begin{array}{l}\text { Medical } \\
\text { (chronic kidney } \\
\text { disease) }\end{array}$ & elf-management & Self-efficacy & Empowerment & $\begin{array}{l}\text { Yes, improvement } \\
\text { in diet, dialysis, } \\
\text { quality of life and } \\
\text { satisfaction with care }\end{array}$ & Yes \\
\hline $\begin{array}{l}\text { Patient satisfaction with } \\
\text { nurse-led telephone } \\
\text { follow-up after curative } \\
\text { treatment for breast } \\
\text { cancer }\end{array}$ & $\begin{array}{c}\text { Kimman et al. } \\
\text { 2010, The } \\
\text { Netherlands } \\
\text { [70] }\end{array}$ & $\begin{array}{c}\text { Oncological } \\
\text { (breast cancer) }\end{array}$ & $\begin{array}{c}\text { Patient } \\
\text { satisfaction }\end{array}$ & Dialogue & Follow-up & $\begin{array}{l}\text { Yes, same level of } \\
\text { patient satisfaction } \\
\text { (compared to } \\
\text { hospital follow-up) }\end{array}$ & No \\
\hline $\begin{array}{l}\text { Nurse-led follow-up } \\
\text { of patients after } \\
\text { oesophageal or gastric } \\
\text { cardia cancer surgery: } \\
\text { a randomised trial }\end{array}$ & $\begin{array}{l}\text { Verschuur et al. } \\
\text { 2009, The } \\
\text { Netherlands } \\
\text { [71] }\end{array}$ & $\begin{array}{l}\text { Oncological } \\
\text { (esophageal or } \\
\text { gastric cancer) }\end{array}$ & QoL & Assessment & Follow-up & $\begin{array}{l}\text { Yes, CNS home visits } \\
\text { as effective as } \\
\text { follow-up by } \\
\text { clinicians (HRQoL) }\end{array}$ & Yes \\
\hline $\begin{array}{l}\text { Do OA patients gain } \\
\text { additional benefit from } \\
\text { care from a clinical } \\
\text { nurse specialist? A RCT }\end{array}$ & $\begin{array}{l}\text { Hill et al. } \\
\text { 2009, UK } \\
\quad[72]\end{array}$ & $\begin{array}{l}\text { Rheumatology } \\
\text { (osteoarthritis) }\end{array}$ & $\begin{array}{c}\text { Patient } \\
\text { satisfaction }\end{array}$ & Assessment & Consultation & $\begin{array}{l}\text { Yes, CNS clinic as } \\
\text { effective as JHD clinic } \\
\text { (health status and } \\
\text { self-efficacy) }\end{array}$ & Yes \\
\hline $\begin{array}{l}{ }^{\mathrm{a}} \text { Impact of a } \\
\text { rheumatology expert } \\
\text { nurse on the wellbeing } \\
\text { of patients attending a } \\
\text { drug monitoring clinic }\end{array}$ & $\begin{array}{c}\text { Ryan et al. } \\
\text { 2006, UK } \\
{[73]}\end{array}$ & $\begin{array}{l}\text { Rheumatology } \\
\text { (osteoarthritis) }\end{array}$ & QoL & Assessment & Consultation & $\begin{array}{c}\text { Yes, improved } \\
\text { wellbeing (health } \\
\text { status and arthritis } \\
\text { control) }\end{array}$ & Yes \\
\hline
\end{tabular}

AMI = Acute Myocardial Infarction, BMI = Body Mass Index, BP = Blood Pressure, CBT = Cognitive Behavioral Therapy, CNS = Clinical Nurse Specialist, COPD = Chronic Obstructive Pulmonary Disease, EBC = Evidence-Based Care, GP = General Practitioner, HRQL = Health-Related Quality of Life, JHD = Junior Hospital Doctor, LOS = Length of hospital Stay, NP = Nurse Practitioner, QoL = Quality of Life, RCT = Randomized Controlled Trial, SBP = Systolic Blood Pressure, ${ }^{*}=$ Significant role of the nurse, ${ }^{\mathrm{a}}=$ Theory-based intervention.

fectiveness compared to the non-theory-based RCTs (6 of 16; 38\%). The RCTs with positive effectiveness showed prominent figures as to patient-centered ambition with regard to goal, nurse-directed and nurse-patientdirected initiatives with regard to strategy, and patient-nurse interaction with regard to content (Table 4). These figures should be compared to RCTs without effectiveness showing more prominent figures at professionalcentered ambition (goal), at nurse-directed initiatives (strategy) and both at patient-nurse interaction and nursing management plans (content). 
Table 2. Theoretical standpoints used in the theory-based studies $(n=26)$.

[24] Jiang et al. 2007, [30] Sisk et al. 2006, [37] Harrison et al. 2008, [40] ter Bogt et al. 2009, [45] Houweling et al. 2009, [57] Willems et al. 2008, [62] Artinian et al. 2007, [63] Goessens et al. 2006, [77] Elley et al. 2008

[31] Smeulders et al. 2010, [44] Hawkins 2010, [64] Sol et al. 2007

[21] Goodman et al. 2008, [44] Hawkins 2010, [65] Tonstad et al. 2007

[38] Lamers et al. 2010a, [52] Lamers et al. 2010b

[32] Zhao and Wong 2009, [69] Wong et al. 2010

[27] Mittag et al. 2006, [36] Espie et al. 2007

[43] Amoako \& Skelly 2007

[22] Hanssen et al. 2007

[26] Malm et al. 2007

[73] Ryan et al. 2006

[67] Chow \& Wong 2010

[28] Shearer et al. 2007
International evidence-based guidelines

9

Bandura's self-efficacy theory

3

The trans-theoretical model of health behavior change

Minimal psychological intervention

The four Cs model: comprehensiveness,

collaboration, coordination, continuity

Cognitive-behavioral and health psychology /Cognitive behavioral therapy

Mishel's uncertainty-in-illness theory

Lazarus and Folkman's theory on stress, appraisal, and coping

Orem's self-care theory

Pendelton's framework

Evidence-based care and motivational interviewing

Empowerment using Roger's science of unitary human being person-environment process

Table 3. Categorization matrix the interventional goal, strategy and content in the studies analysed $(n=55)$.

\begin{tabular}{|c|c|c|c|c|c|}
\hline \multicolumn{2}{|c|}{ Goal } & \multicolumn{2}{|c|}{ Strategy } & \multicolumn{2}{|c|}{ Content } \\
\hline Category & Main category & Category & Main category & Category & Main category \\
\hline $\begin{array}{c}\text { Quality of life (11) } \\
\text { Self-management (9) } \\
\text { Self-care behaviour (5) } \\
\text { Patient satisfaction (3) }\end{array}$ & $\begin{array}{l}\text { Patient-centered } \\
\text { ambition (28) }\end{array}$ & $\begin{array}{c}\text { Video/telemonitoring } \\
\text { (4) } \\
\text { Self-monitoring (3) } \\
\text { Practical training (1) } \\
\text { Dialogue (14) } \\
\text { Self-efficacy (6) } \\
\text { CBT (3) } \\
\text { Listening therapy (1) } \\
\text { Adult learning (1) }\end{array}$ & $\begin{array}{l}\text { Patient-directed } \\
\text { initiatives (8) }\end{array}$ & $\begin{array}{c}\text { Counselling (7) } \\
\text { Support (7) } \\
\text { Empowerment (6) } \\
\text { Advice (6) } \\
\text { Education (2) } \\
\text { Motivation (1) }\end{array}$ & $\begin{array}{l}\text { Patient-nurse } \\
\text { interaction plan } \\
\text { (29) }\end{array}$ \\
\hline $\begin{array}{l}\text { Prevention (15) } \\
\text { Adherence (5) } \\
\text { EBC (5) } \\
\text { Detection (2) }\end{array}$ & $\begin{array}{c}\text { Professional-centered } \\
\text { ambition (27) }\end{array}$ & $\begin{array}{l}\text { Assessment (17) } \\
\text { Care plans (3) } \\
\text { EVB algorithm (2) }\end{array}$ & $\begin{array}{l}\text { Nurse-directed } \\
\text { initiatives (22) }\end{array}$ & $\begin{array}{c}\text { Follow-up/feedback } \\
\text { (10) } \\
\text { Consultation (8) } \\
\text { Screening (3) } \\
\text { Symptom } \\
\text { management (3) } \\
\text { Wound care (2) }\end{array}$ & $\begin{array}{l}\text { Nursing } \\
\text { management plan } \\
\text { (26) }\end{array}$ \\
\hline
\end{tabular}

Table 4. Studies with effect $(n=39)$ and without effect $(n=16)$ in relation to interventional goal, strategy and content.

\begin{tabular}{ccc}
\hline Intervention & Studies with effect, $\mathrm{n}(\%)$ & Studies without effect, $\mathrm{n}(\%)$ \\
\hline Intervention goal & & $7(44)$ \\
Patient-centered ambition & $21(54)$ & $9(56)$ \\
Professional-centered ambition & $18(46)$ & $4(25)$ \\
Intervention strategy & & $5(31)$ \\
Patient-directed initiatives & $10(26)$ & $7(44)$ \\
Nurse-patient directed initiatives & $14(36)$ & $8(50)$ \\
Nurse-directed initiatives & $15(38)$ & $8(50)$ \\
Intervention content & & $21(54)$ \\
Patient-nurse interaction plan & $18(46)$ & \\
Nursing management plan & &
\end{tabular}




\section{Discussion}

\subsection{Methodological Issues}

It is interesting and remarkable that less than one-third of the RCTs identified reached the final review process indicating the importance of setting up inclusion and exclusion criteria as well as quality assessment, even in "the RCT world". At the same time it is of importance to remember that also qualitative designs are essential in nursing to identify patients' needs and desiderata in order to develop the most appropriate and effective PROM interventions [74]. A possible limitation was that only two data bases were screened with regard to nurse-led RCTs; but it is important to stress that these data bases were the most relevant ones-CINAHL and PubMed for the purpose of nurse-led interventions in somatic care. Another possible limitation was to study the phenomenon in just one context; the somatic one. From a methodological standpoint it is essential to handle data in a proper way with a sufficient review competence; in this case it was likely so, as all reviewers were researchers familiar within the somatic care context. Of course it would be of value to compare if, and in what way nurse-led RCTs in somatic care differ from the context of women's, children's and mental health. Another limitation is the extensive review team with a risk of bias in the extraction and interpretation processes; but at the same time the review process was guided by an established study protocol [16] as well as the Cochrane review assumptions [18] implying that every single review was scrutinized by the review team until negotiating consensus was reached. It is also a risk to make a fair and proper decision concerning effectiveness or not, due to the studies' choice of primary outcome and the magnitude of clinical relevance and utility from a nursing perspective. Also to determine the nurse's role was sometimes limited due to scanty descriptions of the nurse-led interventions especially with regard to nurses' functions both in a partial and a holistic perspective.

\subsection{Nursing Care Intervention Issues}

Considering the fact that most of the nurse-led intervention studies have been carried out in Europe and North America, it is questionable how well the results are generalizable outside these areas. On the other hand the need for more nurse-led interventions has been emphasized [7], and this seems particularly true for all countries. It is also striking that two care contexts in somatic care stand out; cardiac care and primary care. Both are typical fields engaging both clinical and academic nurses, and the range of lethal diseases like coronary artery disease is extensively engaging both professional interests and socioeconomic resources [13] while the primary care has an increased responsibility for the public health including the whole flora of symptoms, conditions and diagnoses of acute as well as chronic characters [6]. It is satisfactory that as much as $71 \%$ of the nurse-led RCTs ended up with a beneficial nursing care [13], indicating what already has been pointed out [75]. A good reason why nurse-led interventions are successful is obviously the holistic view of the person, e.g. a person-centered care covering all aspects of the person [76] [77]. RCTs with a person-centered care are showing promising figures [5] [78]. Our review underlines this holistic view of a person as RCTs comprising a patient-centered ambition, patient-directed initiative and patient-nurse interaction plan were more prevalent in the nurse-led RCTs with beneficial nursing care effectiveness compared to those without effectiveness. Also that the nurse's role was of significance in two-thirds of these nurse-led RCTs substantiating that the holistic view, i.e. a person-centered care is of importance and a necessity in order to counsel, support, and follow-up the patient in maintaining health or preventing or recovering from disease [79] [80]. Besides the holistic perspective involving a participating patient in his/her total care situation, the person-centered care also advocates the need for and use of EBP [77]. Accordingly, such reasoning highlights the necessity of using theoretical standpoints when operationalizing the study design by using appropriate measurements in order to establish both relevant and effective outcomes. As our study indicates, the theory-based studies (51\%) brought more effectiveness than the non-theory-based ones (38\%), but the use of theory-based strategies is still premature [77]. A theory-based nurse-led RCT intervention points out the grounds in planning and developing the context, goals, strategies, content as well as the nurse's role related to estimated effectiveness.

\section{Conclusions and Implications}

Nurse-led RCTs with a distinct and clear patient-centered ambition, patient-directed initiative and patient-nurse interaction plan seem to promote beneficial nursing care effectiveness. Also a sound theoretical frame was seen to be of importance when designing a nurse-led RCT. Accordingly, more nursing care interventions in nurse-led 
RCTs are needed and can advantageously and theoretically be based on for example a person-centered care. Basic theoretical perspectives are essential when planning and developing evidence-based practice as well as elucidating the role of the nurse in relation to the estimated effects. Concerning effectiveness of nursing care interventions, more reviews are needed in order to compare nurse-led RCTs in somatic care with mental health as well as women's and children's health.

\section{References}

[1] Pravikoff, D.S., Pierce, S.T. and Tanner, A. (2005) Evidence-Based Practice Readiness Study Supported by Academy Nursing Informatics Expert Panel. Nursing Outlook, 53, 49-50. http://dx.doi.org/10.1016/j.outlook.2004.11.002

[2] Fineout-Overholt, E., Melnyk, B.M. and Schultz, A. (2005) Transforming Health Care from the Inside Out: Advancing Evidence-Based Practice in the 21st Century. Journal of Professional Nursing, 21, 335-344. http://dx.doi.org/10.1016/j.profnurs.2005.10.005

[3] Pravikoff, D.S., Tanner, A.B. and Pierce, S.T. (2005b) Readiness of US Nurses for Evidence-Based Practice. American Journal of Nursing, 105, 40-51. http://dx.doi.org/10.1097/00000446-200509000-00025

[4] Conn, V.S. (2008) Testing Nursing Interventions. Western Journal of Nursing Research, 30, 913-914. http://dx.doi.org/10.1177/0193945908323632

[5] Olsson, L.-E., Jakobsson Ung, E., Swedberg, K. and Ekman, I. (2012) Efficacy of Person-Centered Care as an Intervention in Controlled Trials-A Systematic Review. Journal of Clinical Nursing, 22, 456-465. http://dx.doi.org/10.1111/jocn.12039

[6] Mortenius, H., Marklund, B., Palm, L., Fridlund, B. and Baigi, A. (2012) The Utilization of Knowledge of and Interest in Research and Development among Primary Care Staff by Means of Strategic Communication-A Staff Cohort Study. Journal of Evaluation in Clinical Practice, 18, 768-775. http://dx.doi.org/10.1017/S1463423611000624

[7] Stein, K.F., Sargent, J.T. and Rafaels, N. (2007) Intervention Research: Establishing Fidelity of the Independent Variable in Nursing Clinical Trials. Nursing Research, 56, 54-62. http://dx.doi.org/10.1097/00006199-200701000-00007

[8] Fogg, L. and Gross, D. (2000) Threats to Validity in Randomized Clinical Trials. Research in Nursing \& Health, 23, 79-87. http://dx.doi.org/10.1002/(SICI)1098-240X(200002)23:1<79::AID-NUR9>3.0.CO;2-R

[9] Rios, L.P., Ye, C. and Thabane, L. (2010) Association between Framing of the Research Question Using the PICOT Format and Reporting Quality of Randomized Controlled Trials. BMC Medical Research Methodology, 10, 11. http://dx.doi.org/10.1186/1471-2288-10-11

[10] Fineout-Overholt, E., Melnyk, B.M., Stillwell, S.B. and Williamson, K.M. (2010) Evidence-Based Practice Step by Step: Critical Appraisal of the Evidence: Part I. American Journal of Nursing, 110, 47-52. http://dx.doi.org/10.1097/01.NAJ.0000405063.97774.0e

[11] Bolton, L.B., Donaldson, N.E., Rutledge, D.N., Bennett, C. and Brown, D.S. (2007) The Impact of Nursing Interventions: Overview of Effective Interventions, Outcomes, Measures, and Priorities for Future Research. Medical Care Research and Review, 64, 123S-143S. http://dx.doi.org/10.1177/1077558707299248

[12] Coster, S. and Norman, I. (2009) Cochrane Reviews of Educational and Self-Management Interventions to Guide Nursing Practice: A Review. International Journal of Nursing Studies, 46, 508-528. http://dx.doi.org/10.1016/j.ijnurstu.2008.09.009

[13] Allen, J.K. and Dennison, C.R. (2010) Randomized Trials of Nursing Interventions for Secondary Prevention in Patients with Coronary Artery Disease and Heart Failure: Systematic Review. Journal of Cardiovascular Nursing, 25, 207-220. http://dx.doi.org/10.1097/JCN.0b013e3181cc79be

[14] Forbes, A. (2009) Clinical Intervention Research in Nursing. International Journal of Nursing Studies, 46, 557-568. http://dx.doi.org/10.1016/j.ijnurstu.2008.08.012

[15] Tuckett, A.G. (2005) The Care Encounter: Pondering Caring, Honest Communication and Control. International Journal of Nursing Practice, 11, 77-84. http://dx.doi.org/10.1111/j.1440-172X.2005.00505.x

[16] Swedish Council on Health Technology Assessment (2009) Evaluation and Synthesis of Studies Using Quantitative Methods of Analysis. SBU, Stockholm.

[17] Liberati, A., Altman, D.G., Tetzlatt, J., Mulrow, C., Gotzsche, P.C. and Joannidis, J.P. (2009) The Prisma Statement for Reporting Systematic Reviews and Metaanalyses of Studies That Evaluate Healthcare Interventions: Explanations and Elaborations. BMJ, 339, b2700. http://dx.doi.org/10.1136/bmj.b2700

[18] Higgins, J.P.T. and Green, S. (2011) Cochrane Collaboration Handbook for Systematic Review of Interventions. The Cochrane Collaboration, London.

[19] Andryukhin, A., Frolova, E., Vaes, B. and Degryse, J. (2010) The Impact of a Nurse-Led Care Programme on Events 
and Physical and Psychosocial Parameters in Patients with Heart Failure with Preserved Ejection Fraction: A Randomized Clinical Trial in Primary Care in Russia. European Journal of General Practice, 16, 205-214. http://dx.doi.org/10.3109/13814788.2010.527938

[20] Baig A.A., Mangione, C.M., Sorrell-Thompson, A.L. and Miranda, J.M. (2010) A Randomized Community-Based Intervention Trial Comparing Faith Community Nurse Referrals to Telephone-Assisted Physician Appointments for Health Fair Participants with Elevated Blood Pressure. Journal of General Internal Medicine, 25, 701-709. http://dx.doi.org/10.1007/s11606-010-1326-9

[21] Goodman, H., Parsons, A., Davison, J., Preedy, M., Peters, E., Shuldham, C., et al. (2008) A Randomized Controlled Trial to Evaluate a Nurse-Led Programme of Support and Lifestyle Management for Patients Awaiting Cardiac Surgery "Fit for Surgery: Fit for Life" Study. European Journal of Cardiovascular Nursing, 7, 189-195. http://dx.doi.org/10.1016/j.ejcnurse.2007.11.001

[22] Hanssen, T.A., Nordrehaug, J.E., Eide, G.E. and Hanestad, B.R. (2007) Improving Outcomes after Myocardial Infarction: A Randomized Controlled Trial Evaluating Effects of a Telephone Follow-Up Intervention. European Journal of Cardiovascular Prevention and Rehabilitation, 14, 429-437. http://dx.doi.org/10.1097/HJR.0b013e32801da123

[23] Jaarsma, T., van der Wal, M.H., Lesman-Leegte, I., Luttik, M.L., Hogenhuis, J., Veeger, N.J., et al. (2008) Effect of Moderate or Intensive Disease Management Program on Outcome in Patients with Heart Failure: Coordinating Study Evaluating Outcomes of Advising and Counseling in Heart Failure (COACH). Archives of Internal Medicine, 168, 316-324. http://dx.doi.org/10.1001/archinternmed.2007.83

[24] Jiang, X., Sit, J.W. and Wong, T.K. (2007) A Nurse-Led Cardiac Rehabilitation Programme Improves Health Behaviours and Cardiac Physiological Risk Parameters: Evidence from Chengdu, China. Journal of Clinical Nursing, 16, 1886-1897. http://dx.doi.org/10.1111/j.1365-2702.2007.01838.x

[25] Lapointe, F., Lepage, S., Larrivée, L. and Maheux, P. (2006) Surveillance and Treatment of Dyslipidemia in the PostInfarct Patient: Can a Nurse-Led Management Approach Make a Difference? Canadian Journal of Cardiology, 22, 761-767. http://dx.doi.org/10.1016/S0828-282X(06)70292-6

[26] Malm, D., Karlsson, J.E. and Fridlund, B. (2007) Effects of a Self-Care Program on the Health-Related Quality of Life of Pacemaker Patients: A Nursing Intervention Study. Canadian Journal of Cardiovascular Nursing, 17, 15-26.

[27] Mittag, O., China, C., Hoberg, E., Juers, E., Kolenda, K.D., Richardt, G., et al. (2006) Outcomes of Cardiac Rehabilitation with versus without a Follow-Up Intervention Rendered by Telephone (Luebeck Follow-Up Trial): Overall and Gender-Specific Effects. International Journal of Rehabilitation Research, 29, 295-302. http://dx.doi.org/10.1097/MRR.0b013e328010ba9a

[28] Shearer, N.B., Cisar, N. and Greenberg, E.A. (2007) A Telephone-Delivered Empowerment Intervention with Patients Diagnosed with Heart Failure. Heart Lung, 36, 159-169. http://dx.doi.org/10.1016/j.hrtlng.2006.08.006

[29] Sherrard, H., Struthers, C., Kearns, S.A., Wells, G., Chen, L. and Mesana, T. (2009) Using Technology to Create a Medication Safety Net for Cardiac Surgery Patients: A Nurse-Led Randomized Control Trial. Canadian Journal of Cardiovascular Nursing, 19, 9-15.

[30] Sisk, J.E., Hebert, P.L., Horowitz, C.R., McLaughlin, M.A., Wan, J.J. and Chassin, M.R. (2006) Effects of Nurse Management on the Quality of Heart Failure Care in Minority Communities: A Randomized Trial. Annals of Internal Medicine, 145, 273-283. http://dx.doi.org/10.7326/0003-4819-145-4-200608150-00007

[31] Smeulders, E.S., van Haastregt, J.C., Ambergen, T., Uszko-Lencer, N.H., Janssen-Boyne, J.J., Gorgels, A.P., et al. (2010) Nurse-Led Self-Management Group Programme for Patients with Congestive Heart Failure: Randomized Controlled Trial. Journal of Advanced Nursing, 66, 1487-1499. http://dx.doi.org/10.1111/j.1365-2648.2010.05318.x

[32] Zhao, Y. and Wong, F.K. (2009) Effects of a Postdischarge Transitional Care Programme for Patients with Coronary Heart Disease in China: A Randomised Controlled Trial. Journal of Clinical Nursing, 18, 2444-2455. http://dx.doi.org/10.1111/j.1365-2702.2009.02835.x

[33] Dierick-van Daele, A.T., Metsemakers, J.F., Derckx, E.W., Spreeuwenberg, C. and Vrijhoef, H.J. (2009) Nurse Practitioners Substituting for General Practitioners: Randomized Controlled Trial. Journal of Advanced Nursing, 65, 391-401. http://dx.doi.org/10.1111/j.1365-2648.2008.04888.x

[34] Dumville, J.C., Worthy, G., Bland, J.M., Cullum, N., Dowson, C., Iglesias, C., et al. (2009) Larval Therapy for Leg Ulcers (VenUS II): Randomised Controlled Trial. BMJ, 19, 338:b773. http://dx.doi.org/10.1136/bmj.b773

[35] Elley, C.R, Robertson, M.C., Garrett, S., Kerse, N.M., McKinlay, E., Lawton, B., et al. (2008) Effectiveness of a Fallsand-Fracture Nurse Coordinator to Reduce Falls: A Randomized, Controlled Trial of At-Risk Older Adults. Journal of the American GeriatricSociety, 56, 1383-1389. http://dx.doi.org/10.1111/j.1532-5415.2008.01802.x

[36] Espie, C.A., MacMahon, K.M., Kelly, H.L., Broomfield, N.M., Douglas, N.J., Engleman, H.M., McKinstry, B., Morin, C.M., Walker, A. and Wilson, P. (2007) Randomized Clinical Effectiveness Trial of Nurse-Administered Small-Group Cognitive Behavior Therapy for Persistent Insomnia in General Practice. Sleep, 30, 574-584. 
[37] Harrison, M., Graham, I.D., Lorimer, K., Vandenkerkhof, E., Buchanan, M., Wells, P.S., Brandys, T. and Pierscianowski, T. (2008) Nurse Clinic versus Home Delivery of Evidence-Based Community Leg Ulcer Care: A Randomized Health Services Trial. BMC Health Services Research, 8, 243. http://dx.doi.org/10.1186/1472-6963-8-243

[38] Lamers, F., Jonkers, C.C., Bosma, H., Kempen, G.I., Meijer, J.A., Penninx, B.W., Knottnerus, J.A. and van Eijk, J.T.M. (2010) A Minimal Psychological Intervention in Chronically Ill Elderly Patients with Depression: A Randomized Trial. Psychotherapy and Psychosomatics, 79, 217-226. http://dx.doi.org/10.1159/000313690.

[39] Spice, C.L., Morotti, W., George, S., Dent, T.H., Rose, J., Harris, S. and Gordon, C.J. (2009) The Winchester Falls Project: A Randomised Controlled Trial of Secondary Prevention of Falls in Older People. Age and Ageing, 38, 33-40. http://dx.doi.org/10.1093/ageing/afn192

[40] Ter Bogt, N.C., Bemelmans, W.J., Beltman, F.W., Broer, J., Smit, A.J. and van der Meer, K. (2009) Preventing Weight Gain: One-Year Results of a Randomized Lifestyle Intervention. American Journal of Preventive Medicine, 37, 270-277. http://archinte.jamanetwork.com/article.aspx?articleid=226662

[41] Ulm, K., Huntgeburth, U., Gnahn, H., Briesenick, C., Pürner, K. and Middeke, M. (2010) Effect of an Intensive NurseManaged Medical Care Programme on Ambulatory Blood Pressure in Hypertensive Patients. Archives of Cardiovascular Disease, 103, 142-149. http://dx.doi.org/10.1016/j.acvd.2010.01.006

[42] Wearden, A.J., Dowrick, C., Chew-Graham, C., Bentall, R.P., Morriss, R.K., Peters, S., Riste, L., Richardson, G., Lovell, K. and Dunn, G. (2010) Nurse Led, Home Based Self-Help Treatment for Patients in Primary Care with Chronic Fatigue Syndrome: Randomised Controlled Trial. British Medical Journal, 340, c1777. http://dx.doi.org/10.1136/bmj.c1777

[43] Amoako, E. and Skelly, A. (2007) Managing Uncertainty in Diabetes: An Intervention for Older African American Women. Ethnicity \& Disease, 17, 515-521.

[44] Hawkins, S.Y. (2010) Improving Glycemic Control in Older Adults Using a Videophone Motivational Diabetes SelfManagement Intervention. Research and Theory for Nursing Practice, 24, $217-232$. http://dx.doi.org/10.1891/1541-6577.24.4.217

[45] Houweling, S.T., Kleefstra, N., van Hateren, K.J., Kooy, A., Groenier, K.H., Ten Vergert, E., Meyboom-de Jong, B., Bilo, H.J. and Langerhans Medical Research Group. (2009) Diabetes Specialist Nurse as Main Care Provider for Patients with Type 2 Diabetes. The Netherlands Journal of Medicine, 67, 279-284.

[46] Huizinga, M.M., Gebretsadik, T., Garcia Ulen, C., Shintani, A.K., Michon, S.R., Shackleford, L.O., Wolff, K.L., Brown, A.W., Rothman, R.L. and Elasy, T.A. (2010) Preventing Glycaemic Relapse in Recently Controlled Type 2 Diabetes Patients: A Randomised Controlled Trial. Diabetologia, 53, 832-839. http://dx.doi.org/10.1007/s00125-010-1658-3

[47] Kim, H.S. and Jeong, H.S. (2007) A Nurse Short Message Service by Cellular Phone in Type-2 Diabetic Patients for Six Months. Journal of Clinical Nursing, 16, 1082-1087. http://dx.doi.org/10.1111/j.1365-2702.2007.01698.x

[48] MacMahon Tone, J., Agha, A., Sherlock, M., Finucane, F., Tormey, W. and Thompson, C.J. (2009) An Intensive Nurse-Led, Multi-Interventional Clinic Is More Successful in Achieving Vascular Risk Reduction Targets than Standard Diabetes Care. Irish Journal of Medical Science, 178, 179-186. http://dx.doi.org/10.1007/s11845-009-0316-6

[49] Nesari, M., Zakerimoghadam, M., Rajab, A., Bassampour, S. and Faghihzadeh, S. (2010) Effect of Telephone FollowUp on Adherence to a Diabetes Therapeutic Regimen. Japan Journal of Nursing Science, 7, 121-128. http://dx.doi.org/10.1111/j.1742-7924.2010.00146.x

[50] Stone, R.A., Rao, R.H., Sevick, M.A., Cheng, C., Hough, L.J. and Macpherson, D.S. (2010) Active Care Management Supported by Home Telemonitoring in Veterans with Type 2 Diabetes: The DiaTel Randomized Controlled Trial. Diabetes Care, 33, 478-484. http://dx.doi.org/10.2337/dc09-1012

[51] Antic, N.A., Buchan, C., Esterman, A., Hensley, M., Naughton, M.T., Rowland, S., Williamson, B., Windler, S., Eckermann, S. and Doug McEvoy, R. (2009) A Randomized Controlled Trial of Nurse-Led Care for Symptomatic ModerateSevere Obstructive Sleep Apnea. American Journal of Respiratory and Critical Care Medicine, 179, 501-508. http://dx.doi.org/10.1164/rccm.200810-15580C

[52] Lamers, F., Jonkers, C.C., Bosma, H., Chavannes, N.H., Knottnerus, J.A. and van Eijk, J.T. (2010) Improving Quality of Life in Depressed COPD Patients: Effectiveness of a Minimal Psychological Intervention. COPD: Journal of Chronic Obstructive Pulmonary Disease, 7, 315-322. http://dx.doi.org/10.3109/15412555.2010.510156

[53] Nathan, J.A., Pearce, L., Field, C., Dotesio-Eyres, N., Sharples, L.D., Cafferty, F. and Laroche, C.M. (2006) A Randomized Controlled Trial of Follow-Up of Patients Discharged from the Hospital Following Acute Asthma: Best Performed by Specialist Nurse or Doctor? Chest, 130, 51-57.

[54] Sridhar, M., Taylor, R., Dawson, S., Roberts, N.J. and Partridge, M.R. (2008) A Nurse Led Intermediate Care Package in Patients Who Have Been Hospitalised with an Acute Exacerbation of Chronic Obstructive Pulmonary Disease. Thorax, 63, 194-200. http://dx.doi.org/10.1136/thx.2007.077578 
[55] van der Meer, V., van Stel, H.F., Bakker, M.J., Roldaan, A.C., Assendelft, W.J., Sterk, P.J., Rabe, K.F., Sont, J.K. and the SMASHING (Self-Management of Asthma Supported by Hospitals, ICT, Nurses and General practitioners) Study Group. (2010) Weekly Self-Monitoring and Treatment Adjustment Benefit Patients with Partly Controlled and Uncontrolled Asthma: An Analysis of the SMASHING Study. Respiratory Research, 11, 74. http://dx.doi.org/10.1186/1465-9921-11-74

[56] Vitacca, M., Bianchi, L., Guerra, A., Fracchia, C., Spanevello, A., Balbi, B. and Scalvini, S. (2009) Tele-Assistance in Chronic Respiratory Failure Patients: A Randomised Clinical Trial. European Respiratory Journal, 33, 411-418. http://dx.doi.org/10.1183/09031936.00005608

[57] Willems, D.C., Joore, M.A., Hendriks, J.J., Nieman, F.H., Severens, J.L. and Wouters, E.F. (2008) The Effectiveness of Nurse-Led Telemonitoring of Asthma: Results of a Randomized Controlled Trial. Journal of Evaluation in Clinical Practice, 14, 600-609. http://dx.doi.org/10.1111/j.1365-2753.2007.00936.x

[58] Cuthbertson, B.H., Rattray, J., Campbell, M.K., Gager, M., Roughton, S., Smith, A., et al. (2009) The PRaCTICaL Study of Nurse Led, Intensive Care Follow-Up Programmes for Improving Long Term Outcomes from Critical Illness: A Pragmatic Randomised Controlled Trial. British Medical Journal, 339, b3723. http://dx.doi.org/10.1136/bmj.b4445

[59] Franzén, C., Brulin, C., Stenlund, H. and Björnstig, U. (2009) Injured Road Users’ Health-Related Quality of Life after Telephone Intervention: A Randomised Controlled Trial. Journal of Clinical Nursing, 18, 108-116. http://dx.doi.org/10.1111/j.1365-2702.2008.02436.x

[60] Leimig, R., Gower, G., Thompson, D.A. and Winsett, R.P. (2008) Infection, Rejection, and Hospitalizations in Transplant Recipients Using Telehealth. Progress in Transplantation, 18, 97-102.

[61] Marcantonio, E.R., Bergmann, M.A., Kiely, D.K., Orav, E.J. and Jones, R.N. (2010) Randomized Trial of a Delirium Abatement Program for Postacute Skilled Nursing Facilities. Journal of the American Geriatrics Society, 58, 10191026. http://dx.doi.org/10.1111/j.1532-5415.2010.02871.x

[62] Artinian, N.T., Flack, J.M., Nordstrom, C.K., Hockman, E.M., Washington, O.G., Jen, K.L. and Fathy, M. (2007) Effects of Nurse-Managed Telemonitoring on Blood Pressure at 12-Month Follow-Up among Urban African Americans. Nursing Research, 56, 312-322. http://dx.doi.org/10.1097/01.NNR.0000289501.45284.6e

[63] Goessens, B.M., Visseren, F.L., Sol, B.G., de Man-van Ginkel, J.M., van der Graaf, Y. and the SMART Study Group. (2006) A Randomized, Controlled Trial for Risk Factor Reduction in Patients with Symptomatic Vascular Disease: The Multidisciplinary Vascular Prevention by Nurses Study (VENUS). European Journal of Preventive Cardiology, 13, 996-1003. http://dx.doi.org/10.1097/01.hjr.0000216549.92184.40

[64] Sol, B.G., van der Graaf, Y., van der Bijl, J.J., Goessens, B.M. and Visseren, F.L. (2008) The Role of Self-Efficacy in Vascular Risk Factor Management: A Randomized Controlled Trial. Patient Education and Counseling, 71, 191-197. http://dx.doi.org/10.1016/j.pec.2007.12.005

[65] Tonstad, S., Alm, C.S. and Sandvik, E. (2007) Effect of Nurse Counseling on Metabolic Risk Factors in Patients with Mild Hypertension: A Randomised Controlled Trial. European Journal of Cardiovascular Nursing, 6, 160-164. http://dx.doi.org/10.1016/j.ejcnurse.2006.07.003

[66] Chan, D., Harris, S., Roderick, P., Brown, D. and Patel, P. (2009) A Randomised Controlled Trial of Structured NurseLed Outpatient Clinic Follow-Up for Dyspeptic Patients after Direct Access Gastroscopy. BMC Gastroenterology, 9, 12. http://dx.doi.org/10.1186/1471-230X-9-12

[67] Chow, S. and Wong, F.K. (2010) Health-Related Quality of Life in Patients Undergoing Peritoneal Dialysis: Effects of a Nurse-Led Case Management Programme. Journal of Advanced Nursing, 66, 1780-1792. http://dx.doi.org/10.1111/j.1365-2648.2010.05324.x

[68] Raje, D., Scott, M., Irvine, T., Walshe, M., Mukhtar, H., Oshowo, A. and Ingham Clark, C. (2007) Telephonic Management of Rectal Bleeding in Young Adults: A Prospective Randomized Controlled Trial. Colorectal Disease, 9, 86-89. http://dx.doi.org/10.1111/j.1463-1318.2006.01049.x

[69] Wong, F.K., Chow, S.K. and Chan, T.M. (2010) Evaluation of a Nurse-Led Disease Management Programme for Chronic Kidney Disease: A Randomized Controlled Trial. International Journal of Nursing Studies, 47, 268-278. http://dx.doi.org/10.1016/j.ijnurstu.2009.07.001

[70] Kimman, M.L., Bloebaum, M.M., Dirksen, C.D., Houben, R.M., Lambin, P. and Boersma, L.J. (2010) Patient Satisfaction with Nurse-Led Telephone Follow-Up after Curative Treatment for Breast Cancer. BMC Cancer, 10, 174. http://dx.doi.org/10.1186/1471-2407-10-174

[71] Verschuur, E.M., Steyerberg, E.W., Tilanus, H.W., Polinder, S., Essink-Bot, M.L., Tran, K.T., van der Gaast, A., Stassen, L.P.S., Kuipers, E.J. and Siersema, P.D. (2009) Nurse-Led Follow-Up of Patients after Oesophageal or Gastric Cardia Cancer Surgery: A Randomised Trial. British Journal of Cancer, 100, 70-76. http://dx.doi.org/10.1038/sj.bjc.6604811

[72] Hill, J., Lewis, M. and Bird, H. (2009) Do OA Patients Gain Additional Benefit from Care from a Clinical Nurse Spe- 
cialist?-A Randomized Clinical Trial. Rheumatology, 48, 658-664. http://dx.doi.org/10.1093/rheumatology/kep049

[73] Ryan, S., Hassell, A.B., Lewis, M. and Farrell, A. (2006) Impact of a Rheumatology Expert Nurse on the Wellbeing of Patients Attending a Drug Monitoring Clinic. Journal of Advanced Nursing, 53, 277-286. http://dx.doi.org/10.1111/j.1365-2648.2006.03725.x

[74] Hildon, Z., Allwood, D. and Black, N. (2012) Making Data More Meaningful: Patients’ Views of the Format and Content of Quality Indicators Comparing Health Care Providers. Patient Education and Counseling, 88, 298-304. http://dx.doi.org/10.1016/j.pec.2012.02.006

[75] Gallagher, R. (2010) 57\% of RCTs of Cardiovascular Nursing Interventions Show that They Improve at Least One Outcome for Secondary Prevention Patients; Optimum Intervention Strategy Unclear. Evidence Based Nursing, 13, 126-127. http://dx.doi.org/10.1136/ebn.13.4.126

[76] Zauszniewski, J.A., Bekhet, A. and Haberlein, S. (2012) A Decade of Published Evidence for Psychiatric and Mental Health Nursing Interventions. Online Journal of Issues in Nursing, 17, 8.

[77] Ekman, I., Swedberg, K., Taft, C., Lindseth, A., Norberg, A., Brink, E., et al. (2011) Person-Centered Care-Ready for Prime Time. European Journal of Cardiovascular Nursing, 10, 248-251. http://dx.doi.org/10.1016/j.ejcnurse.2011.06.008

[78] Maisiak, R., Austin, J.S., West, S.G. and Heck, L. (1996) The Effect of Person-Centered Counseling on the Psychological Status of Persons with Systemic Lupus Erythematosus or Rheumatoid Arthritis: A Randomized, Controlled Trial. Arthritis \& Rheumatism, 9, 60-66. http://dx.doi.org/10.1002/art.1790090111

[79] Prilleltensky, I. (2005) Promoting Well-Being: Time for a Paradigm Shift in Health and Human Services. Scandinavian Journal of Public Health, 33, 53-60. http://dx.doi.org/10.1080/14034950510033381

[80] Specht, J.K., Taylor, R. and Bossen, A.L. (2009) Partnering for Care: The Evidence and the Expert. Journal of Gerontological Nursing, 35, 16-22. http://dx.doi.org/10.3928/00989134-20090301-09 